\title{
Effect of Propofol on Colorectal Cancer Angiogenesis and Metastasis
}

\author{
Yubo Li1 ${ }^{*}$, Yanna Pi \\ ${ }^{1}$ Department of General Surgery, Guangzhou First People’s Hospital, Guangzhou, China \\ ${ }^{2}$ Department of Anesthesiology in the Sixth Affiliated Hospital, Sun Yet-Sen University, Guangzhou, China \\ Email: *lyblxy@126.com
}

How to cite this paper: $\mathrm{Li}, \mathrm{Y}$.B. and $\mathrm{Li}$, Y.N. (2021) Effect of Propofol on Colorectal Cancer Angiogenesis and Metastasis. Open Journal of Gastroenterology, 11, 39-46. https://doi.org/10.4236/ojgas.2021.112004

Received: January 22, 2021

Accepted: February 22, 2021

Published: February 25, 2021

Copyright (c) 2021 by author(s) and Scientific Research Publishing Inc. This work is licensed under the Creative Commons Attribution International License (CC BY 4.0).

http://creativecommons.org/licenses/by/4.0/ (c) (i) Open Access

\begin{abstract}
Background: Vascular Endothelial Growth Factor (VEGF) is one of the most critical factors that stimulate angiogenesis in colorectal cancer patients. The Matrix Metalloproteinases (MMPs) are involved in the degeneration of the extracellular matrix and regulation of cell-matrix interactions. MMP-3 plays an important role in metastasis of colorectal cancer. Our aim was to investigate the effect of propofol on VEGF and MMP-3 plasma levels in colorectal cancer patients. Methods: Colorectal cancer patients were included. Blood samples were collected at before and after propofol infusion. VEGF and MMP-3 plasma levels were measured using Enzyme-Linked Immunosorbent Assay (ELISA). Results: A total of 40 colorectal cancer patients were included. The median age of patients was 53 years. Nineteen patients were male and 21 were female. Twenty-five patients were diagnosed with colon cancer and 15 were rectal cancer. The mean propofol infusion dose was $2.02 \mathrm{mg} / \mathrm{kg}$. There were no significant variations in VEGF and MMP-3 plasma levels after propofol infusion compared with before. No side effects occurred in all the patients. Conclusion: Propofol might have no significant effect on VEGF and MMP-3 levels in colorectal cancer patients and further studies need to be investigated.
\end{abstract}

\section{Keywords}

Propofol, VEGF, MMP-3, Colorectal Cancer

\section{Introduction}

Colorectal cancer is one of the most common cancers and one of the most common cancer causes of death globally, accounting for approximately 600,000 deaths per year [1]. Although great progress has been made in the diagnosis and treatment of colorectal cancer, the outcome for these patients is still pessimistic 
for prolonging the survival time and has always been challenging when metastasis occurred [2]. And paradoxically, surgical resection that is the primary treatment of colorectal cancer patients, may directly or indirectly stimulate the growth of residual tumor deposits or circulating tumor cells [3].

Angiogenesis plays an important role in tumor growth and progression in solid tumors. Vascular Endothelial Growth Factor (VEGF) is one of the most critical and specific factors that stimulate both physiological and pathological angiogenesis. Overexpression of VEGF is associated with progression of and poor prognoses for several tumors, especially colorectal cancer [4] [5] [6]. Treatment with a VEGF antagonist significantly attenuated angiogenesis and tumor progress and this treatment has been listed in NCCN guidelines as a molecular therapy for colorectal cancer [7].

The Matrix Metalloproteinases (MMPs) are a family of at least 28 zinc-dependent enzymes which are involved in the degeneration of the Extracellular Matrix (ECM) and regulation of cell-matrix interactions. ECM degradation is a necessary component of metastasis [8]. MMP-3 has been shown to induce EMT in cultured colorectal cancer cells [9] and to play an important role in the growth and/or metastatic transformation of colorectal cancer [10] [11].

Except treatment of surgeries and chemoradiotherapy, anesthetic techniques had been investigated to affect cancer development [12]. Propofol (2,6-diisopropylphenol), one of the most commonly used intravenous anaesthetic agents, also exerts a number of non-anesthetic effects, including anti-tumor activity. Propofol has been attributed with certain anti-inflammatory properties, e.g. via the reduction of inflammatory cytokines [13]. It was also shown that propofol might be able to suppress proliferation and metastasis of colorectal cancer cells [14].

The impact of propofol on VEGF and MMP-3 plasma levels in colorectal cancer patients is unknown. So our aim was to investigate the expression variation of VEGF and MMP-3 after intravenous administration of propofol in colorectal cancer patients.

\section{Methods}

\subsection{Patients}

Patients diagnosed with colorectal cancer, aged $\geq 18$ were eligible for enrollment. Patients with complete or incomplete intestinal obstruction, full stomach, pregnant, allergy to propofol, a history of anesthesia in recent three months or a history of colorectal surgical resection or other type of cancer were excluded. The cases who received chemo- or radio-therapy were excluded. All patients were given informed consent. This clinical trial had been agreed by the ethics committee of hospital.

\subsection{Anaesthesia Management}

The anaesthesia techniques were standardised. No patient received pre-anaesthetic 
medication. Anaesthesia was induced after establishing routine monitoring, including electrocardiogram, non-invasive blood pressure, pulse blood oxygen saturation $\left(\mathrm{SPO}_{2}\right)$, and the Bispectral Index (BIS). All the patients received central venous puncture and catheterization. Propofol was administrated with a speed of $1 \mathrm{mg} / \mathrm{kg} / \mathrm{h}$ until the target BIS value of 50 was achieved (Orchestra ${ }^{\circledR}$ Base Primea; Fresenius Vial, Brezins, France).

\subsection{Blood Sampling and Processing}

To be eligible for entry into this study, venous blood samples were collected in heparin-containing tubes before and after inducing anesthesia through central venous catheter. The samples were processed within $6 \mathrm{~h}$ of collection, and the plasma fraction stored in aliquots at $-80^{\circ} \mathrm{C}$ until the assay was performed.

\subsection{VEGF Measurement}

VEGF levels were measured using a VEGF enzyme-linked immunosorbent assay (ELISA) kit (Invitrogen, Massachusetts, USA). VEGF measurements were performed according to the manufacturer's instructions and quality control was ensured. VEGF concentrations are reported as picograms per milliliter (pg/ml).

\subsection{MMP-3 Measurement}

Plasma levels of total MMP-3 were determined in duplicate using commercially available Enzyme-Linked Immunosorbent Assay (ELISA) (R\&D Systems) according to the manufacturer's instructions. MMP-3 concentrations are reported as nanograms per milliliter ( $\mathrm{ng} / \mathrm{ml})$.

\subsection{Statistical Methods}

All statistical analyses were carried out with SPSS version 17 (SPSS Inc, Chicago, IL, USA). Numerical variables with a normal distribution were presented as the mean \pm standard deviation (SD), and those with a non-normal distribution as the median and interquartile range. A paired t-test or Wilcoxon signed-ranks test was used to compare pre-anesthesia and post-anesthesia index. A P value < 0.05 was considered statistically significant.

\section{Results}

\subsection{Patient Characteristics}

A total of 40 colorectal cancer patients fulfilled inclusion criteria were enrolled in the study after providing informed written consent. The median age of patients was 53 years. Nineteen patients were male and 21 were female. Twenty-five patients were diagnosed with colon cancer and 15 were rectal cancer. Twenty-three patients with primary colorectal cancer were diagnosed at stage II, 14 patients at stage III and three patients with stage IV.

The mean propofol dose was $2.02 \mathrm{mg} / \mathrm{kg}$. There were no side effects occured in all patients, like allergy reactions, severe hypoxemia or other situations need 
emergency measures. Other baseline data had been shown in Table 1.

\subsection{Hemodynamic Index}

The mean blood pressure, $\mathrm{SpO}_{2}$ and hear rate of all the colorectal cancer patients were significantly decreased post-anesthesia compared with pre-anesthesia (Table 2).

\subsection{Serum Expression VEGF and MMP-3 before and Post Anesthesia}

The serological VEGF levels in colorectal cancer patients before anesthesia were $188.70 \pm 22.92 \mathrm{pg} / \mathrm{ml}$ compared to that after anesthesia measured as $191.30 \pm$ $22.66 \mathrm{pg} / \mathrm{ml}$, indicating no significant variation in VEGF expression.

The serological MMP-3 levels in colorectal cancer patients before anesthesia were $22.81 \pm 5.11 \mathrm{ng} / \mathrm{ml}$ compared to that after anesthesia measured as $23.13 \pm$ $4.48 \mathrm{ng} / \mathrm{ml}$, indicating no significant variation in MMP-3 expression.

Table 1. Demographic and clinical characteristics of the study population.

\begin{tabular}{lc}
\multicolumn{1}{c}{ Items } \\
\hline Age, years (mean \pm SD) & $53.38 \pm 8.16$ \\
Sex $(\mathrm{n})$ & $19(47.5 \%)$ \\
$\quad$ Male & $21(52.5 \%)$ \\
Female & $24.15 \pm 2.37$ \\
BMI $\left(\mathrm{kg} / \mathrm{m}^{2}\right)$ & 0 \\
Preoperative chemoradiotherapy $(\mathrm{n})$ & \\
Type of cancer (n) & $25(62.5 \%)$ \\
Colon cancer & $15(37.5 \%)$ \\
Rectal cancer & \\
Cancer stage (n) & $23(57.5 \%)$ \\
Stage II & $14(35 \%)$ \\
Stage III & $3(7.5 \%)$ \\
Stage IV & $2.02 \pm 0.22$ \\
Propofol (mg/kg) & \\
\hline
\end{tabular}

Table 2. Hemodynamic index and serum levels of VEGF and MMP-3 pre- and postanesthesia.

\begin{tabular}{lccc}
\hline \multicolumn{1}{c}{ Items } & Pre-anesthesia & Post-anesthesia & P value \\
\hline Mean blood pressure, mmHg (mean $\pm \mathrm{SD})$ & $90.73 \pm 10.01$ & $74.10 \pm 7.60$ & $<0.001$ \\
$\mathrm{SpO}_{2}, \%($ mean $\pm \mathrm{SD})$ & $98.58 \pm 1.26$ & $92.58 \pm 1.75$ & $<0.001$ \\
Heart rate, n/minute (mean $\pm \mathrm{SD})$ & $79.50 \pm 9.32$ & $65.63 \pm 7.84$ & $<0.001$ \\
VEGF, pg/ml (mean $\pm \mathrm{SD})$ & $188.70 \pm 22.92$ & $191.30 \pm 22.66$ & 0.473 \\
MMP-3, ng/ml (mean $\pm \mathrm{SD})$ & $22.81 \pm 5.11$ & $23.13 \pm 4.48$ & 0.190 \\
\hline
\end{tabular}




\section{Discussion}

In this study, we have not detected any statistical difference in expression of VEGF and MMP-3 plasma levels in colorectal cancer patients after propofol infusion.

Propofol, an extensively used intravenous anesthetic agent during cancer resection surgery, has been confirmed to execute anti-tumor effect on multiple cancers, including Colorectal Cancer (CRC). It had been demonstrated that propofol could inhibit CRC cell proliferation, migration and invasion by up-regulating miR-124-3p.1 and down-regulating AKT3 [14]. Wound healing assay and migration assay demonstrated that propofol has the ability to inhibit epithelial-mesenchymal transition that induced by IL-13 in RKO and SW480 cells through up-regulation of miR-361 and miR-135b and thereafter leads to the inhibition of IL-13/STAT6/ZEB1 signaling pathway [15]. Propofol exposure repressed the NMDAR-CAMKII-ERK pathway to inactivate HIF1a and therefore reduced aerobic glycolysis in colorectal cancer cells and in an in vivo xenograft model [16]. And furthermore, propofol promoted cell apoptosis and inhibited cell proliferation in both Colo205 and SW620 cells, through the down-regulation of HOXA11-AS and up-regulation of let-7i [17].

However, sometimes conflicting data have been published in clinical settings. A retrospective cohort study showed that propofol anesthesia for colorectal cancer surgery is associated with better survival irrespective of tumor-node-metastasis stage compared with desflurane [18]. A clinical study showed there was no significant difference in colorectal cancer patients' immune function with propofol anesthesia compared with sevoflurane [19]. And there were also clinical studies showed that anesthesia with propofol and anesthesia with sevoflurane induced similar inflammatory responses during colorectal cancer surgery [13] [20]. So a Sweden study had been conducting a randomised controlled trial of cancer survival after propofol- or sevoflurane-based anesthesia for cancer surgery, which may give us more information [21].

It has been demonstrated that minimally invasive colorectal resection may increase plasma MMP-3 levels directly via surgical trauma or indirectly due to surgery-associated elevations in TNF- $\alpha$ and IL1. Plasma MMP-3 levels remained significantly elevated from baseline for 4 weeks after minimally invasive colorectal resection for CRC [11]. Increased MMP-3 levels may promote metastases or the growth of residual cancer. But there was little study about the effect of propofol in expression of VEGF and MMP-3 plasma levels in colorectal cancer patients.

And in this study there were no significant differences either. This may be due to the difference of total amount and duration of propofol injected into patients. The total amount might not be enough to cause measurable differences of expression of VEGF and MMP-3, as well as the duration between two blood sample collected was too close. We should follow up these patients longer and detect more times after propofol infusion, and it might be some new findings. Other limitations are that most of our patients were T2 stage, and the sample size is 
rather small. So we should enroll more different stages of colorectal patients in the future.

Other intravenous drugs like lidocaine may decrease the chance of tumor metastasis in colorectal cancer patients [22] [23]. So we might combine different anesthetic drugs to optimizing the anesthetic schemes.

Even though, no significant difference had been detected in expression of VEGF and MMP-3 in colorectal cancer patients after propofol infusion in this study, we could not say that propofol has no benefits on colorectal cancer patients. Further studies are needed to clarify the role of anesthesia, especially propofol in cancer recurrence in colorectal cancer patients.

\section{Acknowledgements}

The authors thank Dr. Tao Xiao for support with the data collecting.

\section{Conflicts of Interest}

The authors declare that there is no conflict of interests in this study.

\section{Author Contributions}

Yubo Li designed the study, collected and analyzed the data and wrote the manuscript. Yanna Pi enrolled the patients, collected and analyzed the data and wrote the manuscript. All authors reviewed the final version of this manuscript and agreed to its submission.

\section{Availability of Data and Material}

Data is available from the authors upon reasonable request.

\section{Ethics Approval}

The study was approved by the Ethics Committee.

\section{References}

[1] Brenner, H., Kloor, M. and Pox, C.P. (2014) Colorectal Cancer. Lancet, 383, 1490 1502. https://doi.org/10.1016/S0140-6736(13)61649-9

[2] Fakih, M.G. (2015) Metastatic Colorectal Cancer: Current State and Future Directions. Journal of Clinical Oncology, 33, 1809-1824.

[3] Neeman, E. and Ben-Eliyahu, S. (2013) Surgery and Stress Promote Cancer Metastasis: New Outlooks on Perioperative Mediating Mechanisms and Immune Involvement. Brain, Behavior, and Immunity, 30, S32-S40.

https://doi.org/10.1016/j.bbi.2012.03.006

[4] Kwak, C., Jin, R.J., Lee, C., Park, M.S. and Lee, S.E. (2002) Thrombospondin-1, Vascular Endothelial Growth Factor Expression and Their Relationship with p53 Status in Prostate Cancer and Benign Prostatic Hyperplasia. BJU International, 89, 303-309. https://doi.org/10.1046/j.1464-4096.2001.01417.x

[5] Toi, M., Inada, K., Suzuki, H. and Tominaga, T. (1995) Tumor Angiogenesis in Breast Cancer: Its Importance as a Prognostic Indicator and the Association with 
Vascular Endothelial Growth Factor Expression. Breast Cancer Research and Treatment, 36, 193-204. https://doi.org/10.1007/BF00666040

[6] El-Assal, O.N., Yamanoi, A., Soda, Y., Yamaguchi, M., Igarashi, M., Yamamoto, A., Nabika, T. and Nagasue, N. (1998) Clinical Significance of Microvessel Density and Vascular Endothelial Growth Factor Expression in Hepatocellular Carcinoma and Surrounding Liver: Possible Involvement of Vascular Endothelial Growth Factor in the Angiogenesis of Cirrhotic Liver. Hepatology, 27, 1554-1562.

https://doi.org/10.1002/hep.510270613

[7] Weis, S.M. and Cheresh, D.A. (2011) Tumor Angiogenesis: Molecular Pathways and Therapeutic Targets. Nature Medicine, 17, 1359-1370.

https://doi.org/10.1038/nm.2537

[8] Orlichenko, L.S. and Radisky, D.C. (2008) Matrix Metalloproteinases Stimulate Epithelial-Mesenchymal Transition during Tumor Development. Clinical \& Experimental Metastasis, 25, 593-600. https://doi.org/10.1007/s10585-008-9143-9

[9] Kapoor, C., Vaidya, S., Wadhwan, V., Kaur, G. and Pathak, A. (2016) Seesaw of Matrix Metalloproteinases (MMPs). Journal of Cancer Research and Therapeutics, 12, 28-35. https://doi.org/10.4103/0973-1482.157337

[10] Zucker, S. and Vacirca, J. (2004) Role of Matrix Metalloproteinases (MMPs) in Colorectal Cancer. Cancer and Metastasis Reviews, 23, 101-117. https://doi.org/10.1023/A:1025867130437

[11] Shantha Kumara, H.M.C, Gaita, D.J., Miyagaki, H., Yan, X.H., Herath, S.A.C., Cekic, V. and Whelan, R.L. (2014) Minimally Invasive Colorectal Resection Is Associated with Significantly Elevated Levels of Plasma Matrix Metalloproteinase 3 (MMP-3) during the First Month after Surgery Which May Promote the Growth of Residual Metastases. Surgical Endoscopy, 28, 3322-3328.

https://doi.org/10.1007/s00464-014-3612-9

[12] Cassinello, F., Prieto, I., del Olmo, M., Rivas, S. and Strichartz, G.R. (2015) Cancer Surgery: How MAY Anesthesia Influence Outcome? Journal of Clinical Anesthesia, 27, 262-272. https://doi.org/10.1016/j.jclinane.2015.02.007

[13] Margarit, S.C., Vasian, H.N., Balla, E., Vesa, S. and Ionescu, D.C. (2014) The Influence of Total Intravenous Anaesthesia and Isoflurane Anaesthesia on Plasma Interleukin-6 and Interleukin-10 Concentrations after Colorectal Surgery for Cancer: A Randomized Controlled Trial. European Journal of Anaesthesiology, 31, 678-684. https://doi.org/10.1097/EJA.0000000000000057

[14] Li, Y.J., Dong, W.J., Yang, H. and Xiao, G.P. (2020) Propofol Suppresses Proliferation and Metastasis of Colorectal Cancer Cells by Regulating miR-124-3p.1/AKT3. Biotechnology Letters, 42, 493-504. https://doi.org/10.1007/s10529-019-02787-y

[15] Xu, K.J., Tao, W.M. and Su, Z. (2018) Propofol Prevents IL-13-Induced Epithelial-Mesenchymal Transition in Human Colorectal Cancer Cells. Cell Biology International, 42, 985-993. https://doi.org/10.1002/cbin.10964

[16] Chen, X.Y., Wu, Q.C., Sun, P.F., Zhao, Y.J., Zhu, M.M. and Miao, C.H. (2018) Propofol Disrupts Aerobic Glycolysis in Colorectal Cancer Cells via Inactivation of the NMDAR-CAMKII-ERK Pathway. Cellular Physiology and Biochemistry, 46, 492-504. https://doi.org/10.1159/000488617

[17] Ren, Y.-L. and Zhang, W. (2020) Propofol Promotes Apoptosis of Colorectal Cancer Cells via Alleviating the Suppression of lncRNA HOXA11-AS on miRNA let-7i. Biochemistry and Cell Biology, 98, 90-98. https://doi.org/10.1139/bcb-2018-0235

[18] Wu, Z.-F., Lee, M.-S., Wong, C.-S., Lu, C.-H., Huang, Y.-S., Lin, K.-T., Lou, Y.-S., Lin, C., Chang, Y.-C. and Lai, H.-C. (2018) Anesthesia Is Associated with Better 
Survival Than Desflurane Anesthesia in Colon Cancer Surgery. Anesthesiology, 129, 932-941. https://doi.org/10.1097/ALN.0000000000002357

[19] Chen, Y.J., Liang, M., Zhu, Y.T. and Zhou, D.C. (2015) The Effect of Propofol and Sevoflurane on the Perioperative Immunity in Patients under Laparoscopic Radical Resection of Colorectal Cancer. National Medical Journal of China, 10, 3440-3444.

[20] Tylman, M., Sarbinowski, R., Bengtson, J.P., Kvarnström, A. and Bengtsson, A. (2011) Inflammatory Response in Patients Undergoing Colorectal Cancer Surgery: The Effect of Two Different Anesthetic Techniques. Minerva Anestesiologica, 77, 275-282.

[21] Enlund, M., Enlund, A., Berglund, A. and Bergkvist, L. (2019) Rationale and Design of the CAN Study: An RCT of Survival after Propofol- or Sevoflurane-Based Anesthesia for Cancer Surgery. Current Pharmaceutical Design, 25, 3028-3033. https://doi.org/10.2174/1381612825666190705184218

[22] Qu, X.F., Yang, L., Shi, Q.Q., Wang, X.F., Wang, D.G. and Wu, G.Y. (2018) Lidocaine Inhibits Proliferation and Induces Apoptosis in Colorectal Cancer Cells by Upregulating mir-520a-3p and Targeting EGFR. Pathology-Research and Practice, 214, 1974-1979. https://doi.org/10.1016/j.prp.2018.09.012

[23] Galoș, E.V., Tat, T.F., Popa, R., Efrimescu, C.I., Finnerty, D., Buggy, D.J., Ionescu, D.C. and Mihu, C.M. (2020) Neutrophil Extracellular Trapping and Angiogenesis Biomarkers after Intravenous or Inhalation Anaesthesia with or without Intravenous Lidocaine for Breast Cancer Surgery: A Prospective, Randomised Trial. British Journal of Anaesthesia, 125, 712-721. https://doi.org/10.1016/j.bja.2020.05.003 